\title{
Orientación estratégica, innovación y resultados en PYMES de nueva creación: el rol del marketing
}

\section{Strategic orientation, innovation and performance in new SMEs: the role of marketing}

\author{
JoRge Gómez Villanueva ${ }^{1}$ \\ JoAN LLONCH ANDREU ${ }^{2}$ \\ JOSEP RIALP CRIADO 3
}

Recibido el 26 de noviembre de 2009, aceptado el 3 de junio de 2010

$\mathrm{N}^{\mathrm{o}}$ de clasificación JEL: M30

DOI: $10.5295 / \mathrm{cdg} .100190$ jg

Reseña bibliográfica: GÓMEZ, J., LLONCH, J. y RIALP, J. (2010): “Orientación estratégica, innovación y resultados en PYMES de nueva creación: el rol del marketing”, Cuadernos de Gestión, Vol. 10, no especial, pp. 85-110, DOI: 10.5295/cdg.100190jg

\section{Resumen:}

A pesar del importante papel de las PYMES de nueva creación en el desarrollo económico, no tenemos constancia de trabajos que hayan abordado de manera simultánea el estudio de la relación entre tres orientaciones estratégicas clave como son la orientación emprendedora $(O E)$, la orientación al mercado $(O M)$ y la orientación al aprendizaje $(O A)$ con la innovación y con el éxito de las PYMES de nueva creación. Los trabajos existentes en la actualidad son de carácter parcial, ya que se limitan a estudiar los efectos de sólo algunas de estas tres orientaciones estratégicas en los resultados de dichas empresas (Li y Atuahene-Gima, 2001; Renko et al., 2009). La presente investigación pretende precisamente cubrir esa laguna, formulando un modelo explicativo de los efectos de las diferentes orientaciones estratégicas en la innovación y en los resultados de las PYMES de nueva creación. Los resultados obtenidos a partir de una muestra de 203 PYMES de nueva creación muestran que existe una relación directa entre la OM y el rendimiento. Asimismo, la OM tiene efectos indirectos en el rendimiento a través de la $O E$, la OA y la Innovación. En cambio, la relación entre la OE y la OA con el rendimiento es solo indirecta, a través de la Innovación (I). En definitiva, se demuestra que el marketing tiene un papel fundamental en el éxito de las PYMES de nueva creación.

\begin{abstract}
:
Although the important role that new SME have for economic development, the relationship among three key strategic orientations as Entrepreneurial orientation (EO), Market orientation (MO) and Learning orientation (LO) with innovation and new SME's success has not been previously analyzed. Present studies on this mater are
\end{abstract}

\footnotetext{
${ }^{1}$ Tecnológico de Monterrey (Campus de Guadalajara).Avenida General Ramón Corona, 2514. 45040 Jalisco (México)

${ }^{2}$ Departament d'Economia de l'Empresa. Facultat d'Economia i Empresa. Universitat Autònoma de Barcelona. 08193, Bellaterra (Barcelona). e-mail: joan.llonch@uab.es

${ }^{3}$ Departament d'Economia de l'Empresa. Facultat d'Economia i Empresa. Universitat Autònoma de Barcelona. 08193, Bellaterra (Barcelona)
} 
partial because they focus in only one or two of these orientations (Li and Atuahene-Gima, 2001; Renko et al., 2009). Thus, the present research aims to fill this gap by developing a model to explain the relationship among EO, MO and LO, innovation and new SME performance and test this model within a sample of new SMEs. Empirical results from a sample of 203 new SMEs show that there is direct relationship between Market orientation (MO) and business performance, and also an indirect relationship among $M O$ and performance through LO, EO and Innovation. However, the relationship between $L O$ and EO with performance is only indirect, through Innovation. In summary, marketing has a critical role on the success of new SMEs.

\section{Palabras clave:}

Orientación estratégica, Orientación al mercado, Innovación, PYMES de nueva creación.

\section{Key Words:}

Strategic orientation, Market orientation, Innovation, New SMEs. 


\section{INTRODUCCIÓN}

Las empresas de nueva creación se enfrentan en sus primeros años de vida a grandes dificultades de supervivencia (Watson et al., 1998), lo que motiva que la mayoría de ellas fracasen y desaparezcan del mercado (Osborne, 1993; Urbano, 2006). Dadas las elevadas pérdidas financieras, económicas y sociales que ello provoca, resulta muy valioso tratar de desarrollar modelos e investigaciones que contribuyan a aumentar el éxito de estas empresas (Laitinen, 1992). En este sentido, en el marco de la Teoría de Creación de Empresas (Entrepreneurship) se han llevado a cabo un gran número de investigaciones para identificar los factores determinantes del éxito de las nuevas empresas, aunque todos estos trabajos se centran básicamente en aspectos relativos a la etapa de gestación de la empresa y no a la de su puesta en marcha (Song et al., 2008).

Una vez la nueva empresa inicia su andadura, la Teoría de la Creación de Empresas cede el protagonismo a la Teoría de los Recursos y Capacidades (TRC), teoría que postula que las ventajas competitivas de una empresa nacen de los activos y de las capacidades diferenciales que esa empresa posee (Barney, 1991; Wernerfelt, 1984). Desde la perspectiva de la TRC, la orientación estratégica de la empresa ha sido considerada una importante capacidad empresarial (Zhou et al., 2005). Por orientación estratégica se entiende la filosofía empresarial adoptada por la organización, es decir, aquel conjunto de valores y creencias en relación al modo de gestionar el negocio que condicionan el logro de unos mejores resultados (Gatignon y Xuereb, 1997).

Por otro lado, la innovación es también una capacidad crítica para competir en el mercado (Hitt et al., 2001), y en entornos tan altamente competitivos como los que existen en la actualidad puede llegar a ser la mejor estrategia para asegurar la supervivencia de la empresa (Rosenbuch et al., 2010).

Algunos autores sugieren que la relación entre la orientación estratégica y los resultados depende del tamaño de la empresa (Keskin, 2006; Pelham, 1999) o de la antigüedad de la misma (Brettel, 2009; Kakati, 2003). Del mismo modo, otros autores sostienen que la innovación, ya sea en producto o en proceso, tiene también unas características diferenciales para el caso de las PYMES (Porter, 1980), especialmente si se trata de PYMES de nueva creación (Rosenbusch et al., 2010). De lo anterior se deduce la singularidad de los efectos que en ellas produce la orientación estratégica o la innovación. A la vista de ello, y dada la importancia que las PYMES de nueva creación tienen para la economía de cualquier país (Carter et al., 1994; Shane y Venkataraman, 2000), la presente investigación se plantea estudiar la relación entre la orientación estratégica, la innovación y los resultados en este tipo concreto de empresas.

Por lo que respecta a la orientación estratégica, nos centraremos en el estudio de tres orientaciones estratégicas que han demostrado ejercer efectos muy relevantes en los resultados organizativos, concretamente, la Orientación al mercado (OM), la Orientación emprendedora (OE) y la Orientación al aprendizaje (OA) (Calatone et al., 2002; Kirca et al., 2005; Wiklund y Sheperd, 2005). Si bien el efecto conjunto de esas tres orientaciones en los resultados ha sido estudiado previamente entre empresas grandes (Hult y Ketchen, 2001; Hult et al., 2004), nunca anteriormente se ha abordado ese estudio entre PYMES de nueva creación. 
Por lo que hace referencia a la innovación, nos basaremos en la innovación de producto, ya que ésta se ha demostrado fundamental para el éxito de la empresa (Li y AtuaheneGima, 2001; Sandvik y Sandvik, 2003).

En resumen, la presente investigación persigue los siguientes objetivos:

a) Formular un modelo teórico que explique la relación entre la orientación estratégica (concretamente, la orientación al mercado, la orientación emprendedora y la orientación al aprendizaje), la innovación y los resultados en las PYMES de nueva creación.

b) Elaborar un conjunto de hipótesis de relación entre las variables de dicho modelo.

c) Poner a prueba el modelo entre una muestra de PYMES de nueva creación.

Al realizar esta investigación seguimos la recomendación de autores que han sugerido recientemente investigar la relación entre las capacidades empresariales y los resultados de las empresas de nueva creación, al considerar que no existen suficientes estudios científicos que hayan abordado esta tarea (Minniti y Lévesque, 2008).

Para lograr dichos objetivos procederemos del siguiente modo: en el siguiente apartado trataremos el marco teórico de la investigación; a continuación presentaremos nuestro modelo y las hipótesis a contrastar; posteriormente, describiremos la metodología y la muestra utilizadas; más adelante expondremos los resultados, y finalmente presentaremos las conclusiones, las limitaciones y las futuras líneas de investigación.

\section{MARCO TEÓRICO: LA TEORÍA DE LOS RECURSOS Y CAPACIDADES}

La Teoría de los Recursos y Capacidades (TRC) se centra en la heterogeneidad y la inmovilidad de los recursos como fuente de ventajas competitivas para la obtención de unos mejores resultados (Barney, 1991). Los recursos de la empresa se pueden clasificar en activos y en capacidades (Day, 1994; Hunt y Morgan, 1995; Wernerfelt, 1984). Los activos son recursos de carácter más tangible que la empresa ha ido acumulando a lo largo del tiempo (por ejemplo, las economías de escala, el prestigio o la imagen de marca), mientras que las capacidades son habilidades más intangibles y más difíciles de cuantificar en términos monetarios (Zhou et al., 2005). Las capacidades consisten básicamente en habilidades que van indisolublemente unidas a las rutinas y a las prácticas de la empresa y que condicionan significativamente sus resultados (Barney, 1991). Las capacidades son heterogéneas entre las firmas, ya que algunas tienen capacidades valiosas que otras no poseen; las que tienen superiores capacidades tendrán también mayores ventajas competitivas y unos mejores resultados (Peteraf, 1993). Para que un recurso empresarial pueda llegar a ser una fuente de ventajas competitivas debe ser raro, valioso y difícil de imitar (Barney, 1991; 2001).

Desde la perspectiva de la TRC, la orientación estratégica de la empresa ha sido considerada una importante capacidad empresarial (Hult y Ketchen, 2001; Zhou et al., 2005). Lo anterior se justifica en el hecho de que la orientación estratégica puede llegar a ser un recurso raro, valioso y difícil de imitar, y convertirse así en una fuente de ventajas competitivas para la empresa (Bhuian et al., 2005; Hult y Ketchen, 2001). Destacan en la literatura tres orientaciones estratégicas que han demostrado ejercer efectos muy relevantes en los resultados organizativos, concretamente, la Orientación al mercado (OM), la Orientación 
emprendedora (OE) y la Orientación al aprendizaje (OA) (Calatone et al., 2002; Kirca et al., 2005; Wiklund y Sheperd, 2005).

La OM es el resultado de la adopción del concepto de marketing en la empresa (Kohli y Jaworski, 1990). La OM se define como "la cultura organizativa que de forma más eficaz y eficiente genera los comportamientos necesarios para la creación de un valor superior para los compradores, y por consiguiente unos mejores resultados para la empresa" (Narver y Slater, 1990, p. 21). Para estar orientada al mercado la empresa debe de orientarse a sus clientes, a sus competidores y presentar una coordinación entre todas sus funciones (Narver y Slater, 1990). Diversos autores han sostenido que la OM es una capacidad clave en cualquier organización empresarial (Bhuian, et al., 2005; Kropp et al., 2006; Hunt y Morgan, 1995).

Por otro lado, la OA es una rutina organizativa de alto nivel que permite generar conocimiento útil para aprovechar las oportunidades del mercado (Sinkula, 1994; Slater y Narver, 1995). A través de dicha rutina organizativa, la empresa se cuestiona de forma proactiva hasta que punto sus creencias y comportamientos actuales contribuyen a maximizar los resultados de la empresa (Baker y Sinkula, 2002). La OA implica la adopción de tres actitudes diferenciadas: compromiso con el aprendizaje, mentalidad abierta y visión compartida (Sinkula et al., 1997). Varias investigaciones recientes han postulado que la OA es una relevante capacidad empresarial (Farrell et al., 2008; Kropp et al., 2006).

$\mathrm{La} \mathrm{OE}$ se define como la capacidad de afrontar los riesgos del entorno, identificar de forma proactiva las oportunidades de desarrollo tecnológico y perseverar en la obtención de los recursos necesarios para el crecimiento de la empresa (Lumpkin y Dess, 2001). La OE refleja el nivel de prioridad que la empresa otorga al proceso de identificar y explotar las oportunidades del mercado (Baker y Sinkula, 2009). La OE se asocia normalmente con tres dimensiones: la innovación, la proactividad y la asunción de riesgos. Numerosos trabajos han considerado también que la $\mathrm{OE}$ es una capacidad empresarial de primer nivel (Bhuian et al., 2005; Runyan et al., 2008; Zhou et al., 2005).

La innovación puede definirse como la habilidad de la organización para adoptar o aplicar de manera exitosa nuevas ideas, procesos o productos (Hurley y Hult, 1998). Conviene no confundir el término innovación ("innovation" o "innovation capacity" en terminología inglesa) con el término "innovativeness". El término "innovativeness" hace referencia a la cultura organizativa que fomenta una actitud de apertura hacia las nuevas ideas (Hult y Ketchen 2001; Verhees y Meulenberg 2004). Por tanto, mientras que la "innovativeness" refleja la postura de la empresa ante el proceso innovador, la innovación reflejaría el output de dicho proceso (Baker y Sinkula, 2009). Como en el presente trabajo pretendemos estudiar las consecuencias de la orientación estratégica en el éxito de las PYMES de nueva creación, tal como hacen también otros autores relevantes (Gatignon y Xuereb, 1997; Li y Atuahene-Gima, 2001; Baker y Sinkula, 2009), adoptaremos el concepto de innovación, es decir el output del proceso innovador, y no el de "innovativeness".

La innovación se clasifica normalmente en innovación de producto y de proceso (Camison-Zornoza et al., 2004). Mientras que la innovación de producto consiste en la introducción de nuevos productos en el mercado (Sandvik y Sandvik, 2003), la de proceso se basa en introducir nuevos elementos, equipos o métodos en la empresa (Camison-Zornoza et al., 2004). Como la introducción de nuevos productos en segmentos atractivos del mercado es una estrategia muy efectiva para las PYMES, ya que les permite evadir la competencia 
(Porter, 1980), en el presente trabajo nos centraremos en el análisis de la innovación de producto y no en la de proceso.

En resumen, dado que las PYMES de nueva creación presentan un elevado riesgo de fracaso (Runyan et al., 2008), y que la innovación es una estrategia crítica para dichas empresas (Rosenbusch et al., 2010), resulta muy importante estudiar la relación entre la orientación estratégica, la innovación y los resultados en el marco de las PYMES de nueva creación (Renko et al., 2009).

\section{DESARROLLO DEL MODELO Y FORMULACIÓN DE HIPÓTESIS}

\subsection{Desarrollo del modelo}

La mayoría de investigaciones que contemplan la relación entre la orientación estratégica, la innovación y los resultados se basan en modelos que consideran el impacto de solo algunas de las orientaciones estratégicas a las que nos referimos anteriormente, es decir, la OM, la OA y la OE (Alvarez et al., 2000; Atuahene-Gima et al., 2005; Baker y Sinkula, 2009; Gatignon y Xurueb, 1997; Paladino, 2007, 2008). Sólo tenemos constancia de un par de investigaciones que hayan estudiado el efecto combinado de esas tres orientaciones estratégicas en la innovación y en los resultados de la empresa (concretamente, Hult y Ketchen, 2001; Hult et al., 2004). Con todo, las dos referidas investigaciones adoptan el concepto de innovación basado en la "innovativeness", y no en la "innovation capacity", que es el concepto que nosotros utilizamos en nuestro trabajo. Además, ninguna de esas investigaciones está pensada específicamente para PYMES de nueva creación, sino para empresas grandes ya establecidas en el mercado. Por tanto, el presente trabajo es el primero en el que se formula un modelo que contempla la relación entre la OM, la OA y la OE, la Innovación, y los resultados para las PYMES de nueva creación.

Con respecto al mencionado modelo, nuestra postura es que en el origen de la estrategia de las PYMES de nueva creación está el conocimiento de mercado, es decir, la OM como un antecedente que guiará tanto a la OA como a la OE. Sostenemos también que la OM tiene un efecto directo en los resultados de las PYMES de nueva creación, así como también un efecto indirecto a través de la OA, la OE y de la Innovación. Por otro lado, postulamos que tanto la OA como la OE influyen directamente en la Innovación, y ésta, a su vez, influye en los resultados organizativos. Por el contrario, partimos de la base de que la relación entre la OM y la Innovación es de carácter indirecto. A continuación formularemos las hipótesis específicas que se derivan del modelo.

\subsection{Formulación de hipótesis}

La mayoría de las evidencias empíricas obtenidas hasta la fecha sugieren la existencia de una relación positiva y directa entre el grado de OM y los resultados organizativos (Ellis, 2006; Kirca et al., 2005). Esa relación directa entre la OM y los resultados se justifica por el valor que dicha orientación añade a la empresa, ya que le ayuda a elaborar un marketing mix enfocado a segmentos específicos del mercado (Baker y Sinkula, 2009). 
En el caso de las PYMES, dada la limitación de recursos que presentan estas empresas y el elevado coste de otras alternativas estratégicas, la OM es la mejor opción para que éstas puedan crear más valor para sus clientes a un coste asumible (Pelham y Wilson, 1996). Con respecto al caso concreto de las PYMES de nueva creación, como son empresas que operan normalmente en entornos muy inciertos y que a menudo carecen de procesos formales de planificación (Pelham, 2000), la OM les permitirá definir mejor sus objetivos y desarrollar mas eficazmente sus actividades en el mercado (Brettel et al., 2009), lo que fomentará que consigan unos mejores resultados (Kakati, 2003). Además, como la OM promueve que las empresas obtengan información sobre las necesidades de los clientes y respondan en consonancia con la misma (Kholi y Jaworski, 1990), la OM ejerce un papel clave en los resultados de las nuevas empresas (Song et al., 2008). A la vista de lo anterior proponemos:

H1. En las PYMES de nueva creación existe una relación positiva y directa entre la orientación al mercado y los resultados de la empresa.

La adopción de una OA propicia que la organización se cuestione continuamente los modelos mentales y las lógicas imperantes en la empresa, contribuyendo así a la creación de conocimiento y al desarrollo de competencias que le permitirán responder mejor a los cambios del entorno (Baker y Sinkula, 1999ª Slater y Narver, 1995), y obtener unos mejores resultados (Baker y Sinkula, 1999b; Calatone et al., 2002; Farrell, 2000). Con todo, para que la organización aprenda y así pueda obtener unos mejores resultados, dado que la complejidad organizativa es mayor en las empresas medianas y grandes que en las pequeñas, la OA (especialmente por lo que respecta al componente "visión compartida") será más importante en las primeras que en las segundas (Wang et al., 2008). A pesar de que la mayoría de evidencias empíricas sobre los efectos de la OA en los resultados se obtuvieron entre empresas grandes (Farrell et al., 2008; Grinstein, 2008), disponemos también de estudios que demuestran la existencia de una relación positiva entre la OA y los resultados tanto en PYMES (Wang, 2008) como en empresas de nueva creación (Kropp et al., 2006), por tanto postulamos que:

H2. En las PYMES de nueva creación existe una relación positiva y directa entre la orientación al aprendizaje y los resultados de la empresa.

Las empresas orientadas al mercado se caracterizan por disponer de una cultura dirigida a la obtención de información sobre el mercado y los clientes (Kohli y Jaworski, 1990). Dicha información les permite crear conocimiento y cuestionarse de forma permanente los valores imperantes en la organización (Slater y Narver, 1995). Por consiguiente, las organizaciones que valoran la importancia de crear conocimiento a partir de disponer de información externa relevante y actualizada (orientándose al mercado), son empresas que se caracterizan también por cuestionar de manera permanente las asunciones de la organización sobre el modo en que funciona el mercado (orientándose al aprendizaje) (Grinstein, 2008; Farrell, 2000). De lo anterior se deduce que la OM contiene el conjunto de valores principales a partir de los cuales se desarrolla la OA (Slater y Narver, 1995). En este sentido, aunque existen algunas posturas que sugieren que la OA es un antecedente de la OM (Mavondo et al., 2005; Santos et al., 2005), la mayoría de investigaciones llegan a la conclusión contraria, es decir, que la OM es el antecedente y la OA la consecuencia, 
tanto en empresas grandes (Farrell, 2000; Jiménez y Cegarra, 2007) como en PYMES (Keskin, 2006; Salavou, 2005). Por lo que respecta a PYMES de nueva creación, a pesar de que no conocemos ninguna investigación empírica que haya abordado el estudio de la relación entre la OM y la OA en este contexto, dado que para que las nuevas empresas puedan utilizar el aprendizaje organizativo para explotar las oportunidades del entorno necesitan primero identificar dichas oportunidades a través de los conocimientos que les otorga la OM (Newbert et al., 2007), la obtención de información sobre el mercado deberá promover en las mismas el desarrollo del aprendizaje organizativo. Por consiguiente proponemos:

H3. En las PYMES de nueva creación existe una relación positiva y directa entre la orientación al mercado y la orientación al aprendizaje.

Las empresas con Orientación Emprendedora se caracterizan por ir siempre unos pasos por delante de la competencia, lo que les permite elegir los mejores nichos de mercado, aplicar precios más altos, y obtener mejores resultados (Zahra y Covin, 1995). Además, las empresas con una $\mathrm{OE}$ tienen la capacidad de responder con rapidez a los cambios del entorno, lo que fomenta la creación de ventajas competitivas y la obtención de unos mejores resultados (Blesa y Ripollés, 2005). Con todo, a pesar que en algunas investigaciones empíricas realizadas entre PYMES o incluso entre PYMES de nueva creación no se obtuvo confirmación a la relación entre la OE y los resultados (Baker y Sinkula, 2009; Stam y Elfring, 2008), en el contexto de las PYMES abundan más las evidencias que demuestran la existencia de una relación positiva entre ambas variables (Keth et al., 2007; Runyan et al., 2008; Wiklund, 1999; Wiklund y Shepherd, 2005). En este sentido, un reciente metaanálisis a cargo Rauch et al., (2009) concluye que sí existe una relación directa y positiva entre la OE y los resultados, y que dicha relación es incluso más intensa entre PYMES que entre grandes empresas. A la vista de ello, y teniendo en cuenta también las evidencias obtenidas entre organizaciones que se encuentran en incubadoras empresariales (Hughes y Morgan, 2007) proponemos:

H4. En las PYMES de nueva creación existe una relación positiva y directa entre la orientación emprendedora y los resultados de la empresa.

La OM es el vehículo adecuado para desarrollar una actividad emprendedora, ya que tanto las empresas orientadas al mercado como las que presentan una orientación emprendedora tratan de satisfacer las necesidades actuales y futuras de los clientes, y expandirse en el mercado aprovechando las oportunidades que éste les ofrece (Grinstein, 2008), por lo que ambas orientaciones son relevantes para alcanzar el éxito de la organización (Bhuian et al., 2005). Con todo, aunque ambas orientaciones puedan adoptarse por separado, una reciente investigación empírica a cargo de Gonzalez-Benito et al., (2009) sugiere que la adopción de una orientación al mercado constituye generalmente el primer paso para la adopción de una orientación emprendedora.

Por otro lado, sabemos que la relación entre la OM y la OE es también clave para el crecimiento y la supervivencia de las PYMES, ya que en esta clase de empresas la combinación de esas dos orientaciones fomenta la adopción de cambios radicales que contribuyen a satisfacer mejor las necesidades actuales y futuras de los clientes (Vázquez et al., 2001). Precisamente, a partir de una investigación empírica entre una muestra muy amplia de 
PYMES, Sciacia et al. (2006) llegan a la conclusión que la OM influye significativamente en la OE.

Aunque no hallamos trabajos empíricos que relacionen la OM y la OE en PYMES de nueva creación, sabemos que la OM es especialmente importante para las organizaciones más emprendedoras y para las de nueva creación, ya que en las fases iniciales de su existencia la OM ayuda a dichas empresas a comprender mejor el entorno, a adaptarse al mismo y a responder de forma rápida a las oportunidades y las amenazas que éste presenta (Grinstein, 2008). A partir de lo anterior postulamos:

H5. En las PYMES de nueva creación existe una relación positiva y directa entre la orientación al mercado y la orientación empresarial.

En el campo del marketing y de la estrategia de empresa se considera como un axioma que el éxito de una innovación es el principal medio a través del cual las empresas consiguen expansionarse en el mercado (Baker y Sinkula, 2009). La innovación de producto acostumbra a influir en las ventas de la empresa ya que generalmente los clientes compran productos que son únicos y que proporcionan un valor superior para los compradores, lo que representa una ventaja sobre los productos de la competencia (Sandvik y Sandvik, 2003).

Cuanto mayor sea el uso de productos innovadores mayor será la ventaja competitiva de la empresa, y mayores dificultades tendrán los competidores para responder de manera efectiva (Sandvik y Sandvik, 2003). Numerosas investigaciones empíricas entre PYMES, al igual que un par de recientes meta-análisis a cargo de Bowen et al., (2009) y de Rosenbusch et al., (2010), han identificado una relación positiva entre la Innovación y los resultados organizativos (Avlonitis y Salavou, 2007; Baker y Sinkula, 2009; Keskin, 2006). Del primero de dichos meta-análisis se sugiere además que la relación entre la Innovación y los resultados es incluso mayor en empresas pequeñas y en empresas de servicios. También se ha demostrado esta misma relación entre empresas de nueva creación (Li y Atuahene-Gima, 2001). Lo anterior nos lleva a formular la siguiente hipótesis:

H6. En las PYMES de nueva creación existe una relación positiva y directa entre la innovación y los resultados de la empresa.

El proceso de aprendizaje organizativo consiste en la adquisición, la diseminación y el uso de conocimiento, y por tanto va íntimamente unido a los resultados de la innovación (Sinkula et al., 1997; Zhou et al., 2005). La OA es una capacidad muy valiosa para una empresa, ya que fomenta que ésta se dedique a entender y a satisfacer las necesidades de sus clientes a través del desarrollo de nuevos productos, nuevos servicios o nuevas formas de hacer negocio (Day, 1994; Slater y Narver, 1995). Asimismo, la OA promueve la generación de un tipo de conocimiento que sirve de base para potenciar el aprendizaje adaptativo, más propio de la OM, y convertirlo en aprendizaje generativo, que es fundamental para el desarrollo de productos, servicios o tecnologías de carácter innovador (Farrell, 2000; Keskin, 2006).

La relación entre la OA y Innovación se ha estudiado ampliamente en los últimos años, encontrándose en la mayoría de casos una relación positiva entre ambas, tanto en empresas grandes (Aragón et al., 2007; Baker y Sinkula, 1999a; Calatone et al., 2002) como también 
en PYMES (Keskin, 2006; Mavondo et al., 2005; Salavou, 2005). Por el contrario, no tenemos constancia de investigaciones que hayan estudiado esta cuestión entre PYMES de nueva creación. Con todo, sabemos que la OA tiene que ver con el desarrollo de conocimiento en la organización (Grinstein, 2008), que la Innovación requiere de un importante nivel de conocimiento (Slater y Narver, 1995) y que la Innovación es una actividad más beneficiosa si cabe para las nuevas empresas que para las ya existentes (Rosenbusch et al., 2010). Las nuevas empresas, al estar todavía en proceso de penetrar en el mercado, tendrán más necesidad de aprender para generar una innovación que les reporte ventajas competitivas. Por tanto, en empresas de nueva creación la OA debería de tener una importante influencia en la innovación, tal como sucede entre empresas que ya llevan tiempo en el mercado. Por consiguiente postulamos:

H7. En las PYMES de nueva creación existe una relación positiva y directa entre la orientación al aprendizaje y innovación.

Los valores propios de una orientación emprendedora fomentan la transformación y la renovación de la empresa, y permiten que ésta adquiera nuevas competencias y desarrolle nuevos modelos de negocio (Grinstein, 2008). Lo anterior contribuye a que la empresa saque mayor provecho de las oportunidades que aparecen en el mercado, y constituye la base para la creación de nuevos productos y para el crecimiento empresarial (Bhuian et al., 2005; Slater y Narver, 1995). Tradicionalmente se ha considerado que existe una estrecha relación entre la OE y la Innovación. Así, por ejemplo, Drucker (1985) sugiere que la innovación es la principal tarea del emprendimiento, o Lumpkin y Dess (1996) sostienen que el énfasis en la innovación es una de las dimensiones clave de la OE. De lo anterior se desprende que el desarrollo de nuevos productos y servicios y la comercialización de los mismos es una importante estrategia emprendedora dirigida a la creación de valor (Hitt et al., 2001; Rauch et al., 2009). A pesar de la estrecha relación existente entre la OE y la Innovación, se trata de dos constructos diferenciados, ya que la OE consiste fundamentalmente en un proceso, mientras que la Innovación es básicamente un resultado (Hult y Ketchen, 2001; Lumpkin y Dess, 1996).

Según diversas investigaciones, la OM también estaría directamente relacionada con la Innovación (Grinstein, 2008; Sandvik y Sandvik, 2003). De todos modos, la relación entre la $\mathrm{OE}$ y la Innovación es normalmente tan intensa que cuando los efectos de la OM y de la OE se contemplan simultáneamente en el mismo modelo el efecto de la OE en la Innovación puede acabar anulando el efecto de la OM en dicha variable (Baker y Sinkula, 2009). Por tanto, dado que en el presente trabajo incluimos en un mismo modelo a la OM y a la $\mathrm{OE}$, es de esperar que la $\mathrm{OE}$ ejerza un efecto directo en la innovación, mientras el efecto de la OM sea sólo indirecto, a través de la propia OE.

Dos recientes investigaciones entre PYMES han demostrado la intensa relación existente entre la OE y la Innovación (Alegre y Chiva, 2009; Baker y Sinkula, 2009). Por otro lado, dado que los esfuerzos del empresario por adoptar actitudes creativas y sin temor al riesgo fomentan la innovación (Kakati, 2003) proponemos que:

H8. En las PYMES de nueva creación existe una relación positiva y directa entre la orientación empresarial y la innovación. 
Figura 1

Modelo propuesto con hipótesis

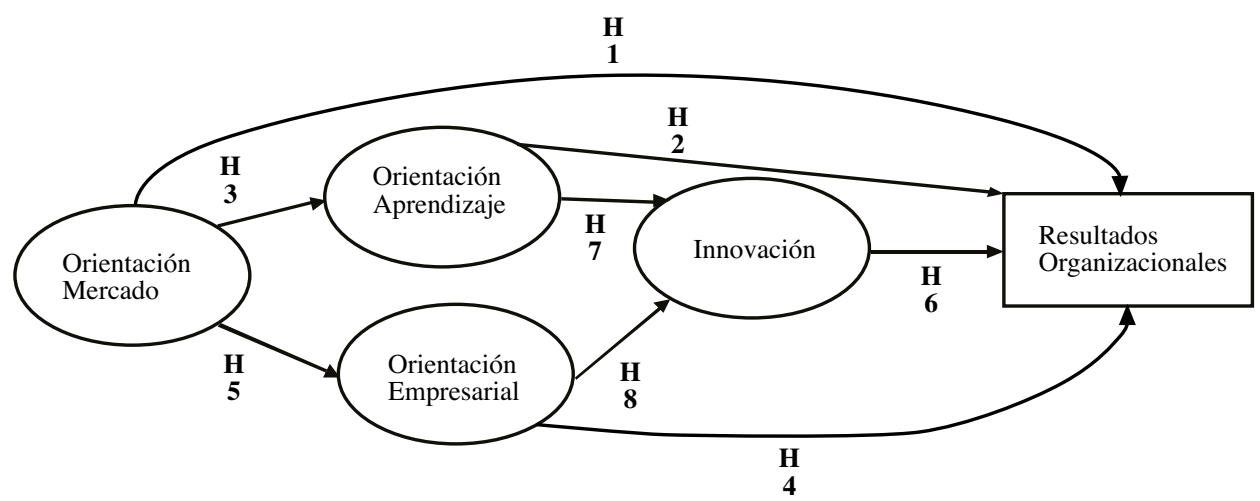

En la figura 1 podemos ver una representación gráfica del modelo, así como de las distintas hipótesis propuestas en relación al mismo.

\section{METODOLOGÍA}

Se ha utilizado la encuesta como método de recolección de información. La muestra utilizada se compone de empresas del sector turístico, específicamente hoteles y restaurantes. Los principales motivos que nos llevaron a seleccionar este sector fueron: a) La importancia de dicho sector, tanto a nivel mundial como especialmente para España, que es un país eminentemente turístico (DIRCE; Haber y Reichel, 2005); b) La gran cantidad de nuevas empresas creadas en este sector en España durante el período de estudio, lo que nos debería permitir trabajar con una muestra con un número elevado de casos (ver tabla 2); y c) El que se trate de un sector compuesto sobre todo por PYMES, y en el que alrededor del 40\% del empleo es autoempleo de los propios emprendedores (Haber y Reichel, 2005), lo que encaja con nuestro objetivo de estudiar PYMES de nueva creación con un elevado componente emprendedor.

Tabla 1

Características de la muestra de empresas

\begin{tabular}{ll|lc|ll}
\hline \multicolumn{2}{r|}{ Tipo de empresa } & \multicolumn{2}{r|}{ Número de empleados } & & Años de antigüedad \\
\hline Hotel & $37 \%$ & -20 & $62 \%$ & 1 & $11 \%$ \\
Restaurante & $63 \%$ & $21-50$ & $30 \%$ & 2 & $26 \%$ \\
& & $>50$ & $8 \%$ & 3 & $19 \%$ \\
& & & 4 & $23 \%$ \\
& & & 5 & $21 \%$ \\
\hline
\end{tabular}


De la base de datos SABI para empresas españolas, se seleccionaron las PYMES que se establecieron entre los años 2001 y 2006, con 10 o más empleados y hasta un máximo de 250 empleados. Las características de la muestra se presentan en la tabla 1, mientras que la tabla 2 ofrece la ficha técnica de la investigación.

Tabla 2

Ficha técnica

\begin{tabular}{l|l}
\hline Población & $\begin{array}{l}\text { Pymes españolas de reciente creación del sector de la hostelería } \\
\text { (entre 2001 y 2006) }\end{array}$ \\
\hline Ámbito & Nacional \\
\hline Método de recolección de datos & Encuesta postal \\
\hline Respuesta a los cuestionarios & Director / Gerente General \\
\hline Tamaño de la población & 916 empresas \\
\hline Tamaño de la muestra & 203 empresas \\
\hline Tasa de respuesta & $22,16 \%$ \\
\hline Error muestral & $6,2 \%$ \\
\hline Nivel de confianza & $95 \%$ z=1,96 p=q=0.5 \\
\hline Trabajo de campo & $\begin{array}{l}\text { Pretest: Septiembre 2006; Primer envío: Octubre 2006; } \\
\text { Segundo envío: Noviembre 2006; Tercer envío: Febrero 2007 }\end{array}$ \\
\hline
\end{tabular}

Para medir las diferentes variables se utilizaron escalas tipo Likert de 7 posiciones (donde 1 significa totalmente en desacuerdo y 7 totalmente de acuerdo). La Orientación al Mercado se midió con la escala MKTOR (Narver y Slater, 1990), con tres componentes: Orientación al Cliente, Orientación Competencia y Coordinación Interfuncional (ver anexo 1). La Orientación al Aprendizaje con una escala sugerida por Baker y Sinkula, (1999a), que se compone de: Compromiso con el Aprendizaje, Mentalidad Abierta y Visión Compartida (ver anexo 2). La Orientación Emprendedora con una escala adaptada de Naman y Slevin (1993) (ver anexo 3). Para medir la Innovación se adaptó una escala sugerida por Baker y Sinkula, (1999) (ver anexo 4). Finalmente, los Resultados Organizativos se midieron de manera subjetiva a partir de un constructo compuesto por 6 ítems: rentabilidad sobre la inversión (ROI), beneficios, incremento de las ventas, grado de satisfacción de los clientes, grado de satisfacción de los empleados y resultados globales. Se solicitó evaluar dichos resultados en comparación con los objetivos de la empresa durante el último ejercicio económico (ver anexo 5).

Para el estudio de la fiabilidad se realizaron los siguientes análisis: (a) una comparación de medias en que se comprobó que no existían diferencias significativas en los resultados obtenidos en los distintos envíos realizados (Armstrong y Overton, 1977); (b) un análisis factorial exploratorio y confirmatorio, en que se constató la consistencia de los componentes de las escalas de OM, OA, OE, Innovación y de Resultados; (c) la fiabilidad de las escalas, que en todos los casos ofrecieron coeficientes $\alpha$ de Cronbach superiores a 0.70, 
que es el mínimo recomendado en la literatura. Asimismo, los valores ítem-to-total correlation para cada una de las escalas tampoco fueron en ningún caso inferiores a 0.70 (véase los anexos 6 y 7).

Para evaluar la validez convergente de las escalas de OM y OA realizamos una análisis parcial con ambos constructos, utilizando la metodología del análisis factorial confirmatorio. El análisis de la validez convergente para las escalas de OE, Innovación y Resultados, como sólo tienen un componente, se realizó a través de la matriz de correlaciones, encontrando una correlación significativa para los tres constructos a niveles de significación de 0.01. Para determinar la validez discriminante se comparó la bondad del ajuste de dos modelos de medida para la escala de OM y de OA, uno que considera una correlación perfecta entre los tres componentes (modelo con restricción), y el otro que no considera esta restricción (modelo sin restricción). Los resultados indicaron con claridad las diferencias a favor del modelo sin restricción en cada uno de los parámetros de medidas de ajuste.

\section{RESULTADOS DEL MODELO PROPUESTO}

La técnica utilizada para contrastar las hipótesis fue la de modelos de ecuaciones estructurales. Las características del modelo se presentan en la figura 2. Los resultados del modelo propuesto muestran parámetros de ajuste adecuados: $\chi^{2}$ normalizada $=1.02$, RM$\mathrm{SEA}=0.01, \mathrm{GFI}=0.89$ y $\mathrm{CFI}=0.99$. La tabla 3 muestra la relación entre las variables del modelo.

Tabla 3

Relación entre variables del modelo propuesto

\begin{tabular}{l|c|c|c|c}
\hline & Estimado & S.E. & P & \\
\hline OM - RO & 0.731 & 0.224 & $* * *$ & H1 (contrastada) \\
\hline OA - RO & 0.104 & 0.214 & n.s. & H2 (no contrastada) \\
\hline OM -> OA & 0.811 & 0.103 & $* * *$ & H3 (contrastada) \\
\hline OE - > RO & 0.072 & 0.213 & n.s. & H4 (no contrastada) \\
\hline OM -> OE & 0.714 & 0.120 & $* * *$ & H5 (contrastada) \\
\hline I -> RO & 0.272 & 0.149 & $* * *$ & H6 (contrastada) \\
\hline OA -> I & 0.168 & 0.107 & $* * *$ & H7 (contrastada) \\
\hline OE -> I & 0.695 & 0.118 & $* * *$ & H8 (contrastada) \\
\hline
\end{tabular}

$* * * \mathrm{p}<.001, \mathrm{n} . \mathrm{s} .=$ no significativa

Observamos en la tabla 3 que la relación entre la OM y los Resultados es positiva de 0.73 , por lo que podemos aceptar la hipótesis 1 . En cambio, la hipótesis 2 no queda contrastada, por lo que no podemos confirmar la influencia directa de la OA en los Resultados. Si se acepta la hipótesis 3 , con el valor positivo y significativo del coeficiente de la relación de 
0.811. Por tanto, la OM es un antecedente de la OA en las PYMES de nueva creación. Por el contrario, no se acepta la hipótesis 4, es decir, la relación directa entre OE y Resultados. Por otro lado, el modelo propuesto presenta una relación positiva y significativa de 0.714 entre la OM y la OE. Por tanto, se confirma la hipótesis 5. Queda contrastada asimismo la hipótesis 6, ya que el valor del coeficiente de relación entre la Innovación (I) y los Resultados es de 0.272 .

Figura 2

\section{Modelo propuesto para ecuaciones estructurales}

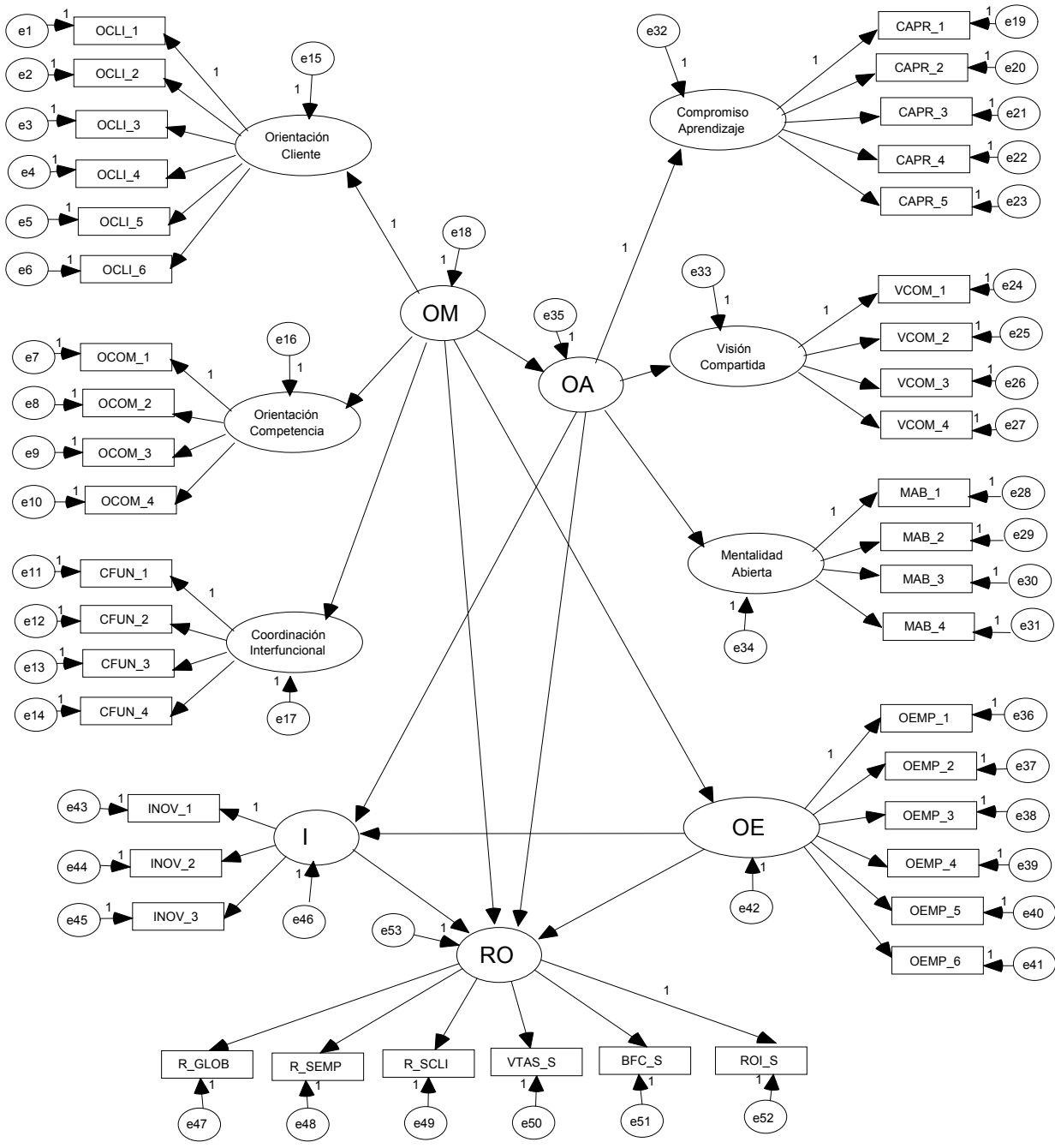


Además, el valor positivo y significativo del coeficiente de relación entre la OA y la Innovación es 0.168 , por tanto, se acepta la hipótesis 7 . Finalmente, el valor positivo y significativo de 0.695 entre la OE y la Innovación indica que la OE es un antecedente de la Innovación, por lo que queda contrastada también la hipótesis 8.

\section{CONCLUSIONES}

La conclusión más destacada del presente trabajo es que la OM se encuentra en el origen de la estrategia de las PYMES de nueva creación para el logro de unos mejores resultados. Como vimos, en este tipo de empresas la OM tiene tanto un efecto directo en los resultados como un efecto indirecto a través de la OA, la OE y la Innovación. Por consiguiente, en estas organizaciones la OM es un antecedente de la OA y de la OE. Contrariamente a lo que pueda suceder en empresas que llevan más tiempo funcionando (Mavondo et al., 2005; Santos et al., 2005), en el caso de las empresas de nueva creación la OM potencia la OA. Tiene sentido que ello sea así ya que para que las nuevas empresas puedan utilizar el aprendizaje organizativo para explotar las oportunidades del entorno necesitan primero identificar dichas oportunidades a través de los conocimientos que les otorga la OM (Newbert et al., 2007). Asimismo, el carácter de antecedente de la OM sobre la OE se justifica en que resulta factible la existencia de una organización con una fuerte orientación emprendedora que no esté orientada al mercado, pero resulta difícil de imaginar una empresa orientada al mercado que no presente un elevado carácter emprendedor (Baker y Sinkula, 2009; Gonzalez-Benito et al., 2009).

Otra conclusión relevante de nuestro trabajo es que la Innovación influye directamente en la obtención de unos mejores resultados. Lo anterior confirmaría las conclusiones de los recientes meta-análisis de Bowen et al. (2009) y de Rosenbuch et al. (2010), en el sentido que la Innovación es especialmente importante para las PYMES. Además, la presente investigación, al trabajar con una muestra de PYMES del sector de hostelería, aportaría asimismo nuevas evidencias sobre la importancia de la innovación en el sector de los servicios, tal como sugieren también Bowen et al. (2009). A partir de los resultados de nuestro trabajo podemos añadir que ello es también cierto cuando se trata de empresas de nueva creación. Por tanto, contrariamente a las conclusiones del meta-análisis realizado por Song et al. (2008) entre nuevas empresas de alta tecnología, los resultados de nuestra investigación sugieren que para el caso de empresas de nueva creación y baja tecnología la Innovación sí influye directamente en los resultados. Así pues, la presente investigación es pionera en demostrar empíricamente la relación existente entre la Innovación y los resultados organizativos entre este tipo de empresas.

Por otro lado, si bien la OM ejerce una relación directa sobre los resultados de la empresa, con respecto a las otras dos orientaciones estudiadas, es decir, la OA y la OE, y contrariamente a las hipótesis formuladas previamente, nuestra investigación sugiere que ninguna de esas dos orientaciones ejerce una relación directa sobre los resultados de las PYMES de nueva creación, sino únicamente una relación indirecta, a través de la Innovación. Con todo, en este tipo de empresas la OE parece tener un efecto más intenso en la Innovación que la OA. Ello se justificaría en el hecho de que las nuevas empresas acostumbran a realizar innovaciones más radicales que las empresas ya existentes (Brettel et 
al., 2009; Rosenbuch et al., 2010), y dado que la OE influye especialmente en el desarrollo de productos realmente innovadores (Baker y Sinkula, 2009), en las nuevas empresas la relación entre la OE-Innovación sería más intensa que la relación OA-Innovación.

Por lo que hace referencia a la ausencia de una relación directa entre la OA y los resultados, las evidencias obtenidas en el presente trabajo confirmarían que la OM es más importante que la OA a la hora de condicionar los resultados de la empresa (Farrell, et al., 2008; Farrell y Oczkowski, 2002), y que ello es de aplicación también para el caso de las PYMES de nueva creación. Para que la OA produzca un efecto directo en los resultados hace falta normalmente que los esfuerzos para que la organización aprenda sean canalizados de manera efectiva hacia la consecución de unos objetivos comunes para toda la empresa (Wang et al., 2008). En el caso de las empresas de nueva creación, dada la importancia que la innovación tiene en las mismas (Brettel et al., 2009; Rosenbusch et al., 2010), la innovación sería un objetivo común que serviría para canalizar los esfuerzos del aprendizaje organizativo y permitiría el logro de unos mejores resultados.

En cuanto a la relación EO-Resultados, el hecho de que no hayamos podido demostrar la existencia de una relación directa entre dichas variables puede justificarse en la elevada variabilidad existente en la relación entre la $\mathrm{OE}$ y los Resultados en función de la investigación de que se trate (Rauch et al., 2009). De hecho, un par de investigaciones previas entre empresas de nueva creación llegaron a una conclusión parecida a la nuestra (Lee et al., 2001; Stam y Elfring, 2008). Ambas investigaciones ofrecen explicaciones a esta cuestión que podrían ser aplicables a nuestro caso. Por ejemplo, Lee et al., (2001) sostienen que ello se debería a la existencia de un desfase temporal de al menos dos años entre los efectos de la OE y los Resultados organizativos, y por tanto, en empresas de nueva creación harían falta al menos dos años para que la OE tuviera un efecto en los Resultados. Por su parte, Stam y Elfring (2008) sugieren que a menudo las empresas de nueva creación no consiguen que la $\mathrm{OE}$ se traduzca en la obtención de unos mejores resultados debido a que normalmente estas empresas carecen de los recursos estratégicos necesarios para que ello se produzca.

En resumen, dado que la OM significa la adopción del concepto de marketing en la empresa, una conclusión clave del presente trabajo es que el marketing tiene un papel fundamental en las PYMES de nueva creación. Así pues, el éxito de estas PYMES pasa en gran medida por orientar la empresa a los clientes y conocer sus necesidades actuales y futuras; por conocer los puntos fuertes y débiles de la competencia y responder de forma rápida a sus acciones; y por mantener una permanente coordinación entre todos los departamentos y funciones, con el objetivo de satisfacer a los clientes de manera continuada y así alcanzar el éxito de la organización.

Asimismo, de esta investigación se extraen algunas implicaciones importantes para la gestión de las empresas. En primer lugar, y por encima de cualquier otra cosa, recomendar que los gestores de las PYMES de reciente creación hagan un gran esfuerzo por orientar la empresa al mercado. Vimos que ello es especialmente importante cuando se trata de empresas pequeñas y del sector de servicios. A través de ese esfuerzo, la empresa estará en disposición de identificar las necesidades y las expectativas de los clientes, conocer las acciones futuras de los competidores y las tendencias del entorno; todo ello con el propósito de identificar nuevas oportunidades de negocio, aspectos que resultan cruciales para este tipo de empresas. 
Igualmente, subrayar la importancia que tiene la Innovación para el logro de unos mejores resultados en este tipo de empresas. Si las PYMES de nueva creación, aunque pertenezcan a sectores de baja tecnología como los hoteles o restaurantes, son capaces de desarrollar ofertas de producto innovadoras tendrán muchas más posibilidades de obtener unos mejores resultados y asegurar así su supervivencia en el mercado.

Por otro lado, a nivel de las políticas públicas, señalar la importancia que puede tener una adecuada formación en marketing para los empresarios que deseen crear su nuevo negocio. Por tanto, si los poderes públicos están interesados en fomentar, no sólo el espíritu emprendedor, sino también y de modo muy especial, el éxito de las iniciativas empresariales, resulta conveniente que otorguen una importancia clave a la formación en marketing en los cursos que se organicen para formar a los nuevos emprendedores. Así pues, los conocimientos de marketing deberían ser una materia básica en cualquier curso de creación de empresas.

Por otro lado, esta investigación presenta algunas limitaciones que hay que contemplar como oportunidades para la investigación futura. En primer lugar, el estudio se basa en una metodología de tipo transversal, mientras que a efectos de extraer conclusiones de tipo causal hubiera sido preferible realizar un estudio de carácter longitudinal. Por otro lado, con respecto a los resultados, sólo tuvimos en cuenta los resultados pasados de la empresa. En este sentido, a efectos de evaluar las consecuencias de la innovación, algunos autores han sugerido tener en cuenta también los resultados futuros (Bowen et al., 2009). Con respecto a la muestra hay que destacar que se ha utilizado una muestra de empresas de un solo sector. Para que los resultados fueran más generalizables sería conveniente replicar la investigación con una muestra más diversificada de PYMES de nueva creación. Otra limitación de nuestro trabajo reside en el hecho de que no se haya tenido en cuenta la influencia del entorno en las relaciones estudiadas. En este sentido, anteriores investigaciones han sugerido la importancia de considerar el entorno a la hora de analizar los efectos de las capacidades empresariales en los resultados organizativos (Hult et al., 2004; Paladino, 2008).

\section{REFERENCIAS BIBLIOGRÁFICAS}

ALEGRE, J. Y CHIVA, R. (2009): "Entrepreneurial orientation, organizational learning capability and performance in the ceramic tiles industry", Instituto Valenciano de Investigaciones Económicas, Working paper, Junio.

ÁLVAREZ, L., SANTOS, M. Y VÁZQUEZ, R. (2000): “Análisis cultural y operativo de la orientación al mercado. Efectos moderadores en la relación OM-Resultados", Revista Española de Investigación en Marketing ESIC, Vol. 4, № 1, pp. 7-41.

ARAGÓN, J.A., GARCÍA, V.J. Y CORDÓN, E. (2007): "Leadership and organizational learning's role on innovation and performance: lessons from Spain", Industrial Marketing Management, Vol. 36, pp. 349-59.

ARMSTRONG, J.S. Y OVERTON, T.S. (1977): "Estimating nonresponse bias in mail surveys", Journal of Marketing Research, Vol. 16 (Agosto), pp. 396-402.

ATUAHENE-GIMA K., SLATER, S.F. Y OLSON, E.M. (2005): "The contingent value of responsive and proactive market orientations for new product program performance", The Journal of Product Innovation Management, Vol. 22, pp. 464-482.

AVLONITIS, G.J. Y SALAVOU, H. (2007): "Entrepreneurial orientation of SMEs, product innovativeness, and performance", Journal of Business Research, Vol. 60, pp. 566-75. 
BAKER, W.E. Y SINKULA, J.M. (1999a): “The synergistic effect of market orientation and learning orientation on organizational performance", Journal of the Academy of Marketing Science, Vol. 27, No. 4, pp. 411-27.

BAKER, W.E. Y SINKULA, J.M. (1999'): "Learning orientation, market orientation, and innovation: integrating and extending models of organizational performance", Journal of Market Focused Management, Vol. 4, No. 4, pp. 295-308.

BAKER, W.E. Y SINKULA, J.M. (2002): "Market orientation, learning orientation and product innovation: delving into the organization's black box", Journal of Market-Focused Management, Vol. 5, pp. 5-23.

BAKER, W.E. Y SINKULA, J.M. (2009): "The complementary effects of market orientation and entrepreneurial orientation on profitability in small businesses", Journal of Small Business Management, Vol. 47, $\mathrm{N}^{\circ}$ 4, pp. 443-464.

BARNEY, J. (1991): "Firm resources and sustained competitive advantage", Journal of Management, Vol. 17, No 1, pp. 99-120.

BARNEY, J. (2001): "Resource-based theories of competitive advantage: A ten year retrospective on the resource-based view", Journal of Management, Vol. 27, $\mathrm{N}^{\circ}$ 6, pp. 643-650.

BLESA, A. Y RIPOLLÉS, M. (2005): "Relación entre la orientación al mercado y la orientación emprendedora: su influencia en el rendimiento de la empresa", Revista Europea de Dirección y Economía de la Empresa, Vol. 14, № 3, pp. 165-80.

BHUIAN, S.N., MENGUC, B. Y BELL, S.J. (2005): "Just entrepreneurial enough: the moderating effect of entrepreneurship on the relationship between market orientation and performance", Journal of Business Research, Vol. 58, pp. 9-17.

BOWEN, F.E., ROSTAMI, M. Y STEEL, P. (2009): “Timing is everthing: a meta-analysis of the relationship between organizational performance and innovation", Journal of Business Research (en prensa).

BRETTEL, M., ENGELEN, A. Y HEINEMANN, F. (2009): "New entrepreneurial ventures in a globalized world: The role of market orientation", Journal of International Entrepreneurship, Vol. 7, pp. 88-110.

CALATONE, R. J., CAVUSGIL, S.T. Y ZHAO, Y. (2002): "Learning orientation, firm innovation capability, and firm performance", Industrial Marketing Management, Vol. 31, pp. 515-524.

CAMISÓN-ZORNOZA, C., LAPIEDRA-ALCAMÍ, R., MERCEDES SEGARRA-CIPRÉS, M. Y BORONAT-NAVARRO, M. (2004): "A meta-analysis of innovation and organizational size", Organization Studies, Vol. 25, № 3, pp. 331-361

CARTER, N.M., STEARNS, T.M., REYNOLDS, P.D. Y MILLER, B.A. (1994): "New venture strategies: theory development with an empirical base", Strategic Management Journal, Vol. $15, \mathrm{~N}^{\mathrm{o}} 1$, pp. 21-41.

DAY, G.S. (1994): "The capabilities of market-driven organizations", Journal of Marketing, Vol. 58, pp. 37-52.

DRUCKER, P. (1985): Innovation and Entrepreneurship, Harper Collins, NY.

ELLIS, P.D. (2006): "Market orientation and performance: a meta-analysis and cross-national comparisons", Journal of Management Studies, Vol. 43, № 5, pp. 1089-1107.

FARRELL, M.A. (2000): "Developing a market-oriented learning organisation", Australian Journal of Management, (Septiembre), pp. 201-223.

FARRELL, M.A. Y OCZKOWSKI, E. (2002): "Are market orientation and learning orientation necessary for superior organizational performance?" Journal of Market-Focused Management, Vol. 5, pp. 197-217.

FARRELL, M.A., OCZKOWSKI, E. Y KHARABSHEH, R. (2008): "Market orientation, learning orientation and organisational performance in international joint ventures", Asia Pacific Journal of Marketing and Logistics, Vol. 20, №. 3, pp. 289-308. 
GATIGNON, H. Y XURUEB, J.M. (1997): "Strategic orientation of the firm and new product performance", Journal of Marketing Research, Vol. 34, pp. 77-90.

GONZÁLEZ-BENITO, O., GONZÁLEZ-BENITO, J Y MUÑOZ-GALLEGO, P.A. (2009): "Role of entrepreneurship and market orientation in firms' success", European Journal of Marketing, Vol. $43 \mathrm{~N}^{\circ} 3 / 4$, pp. $500-522$.

GRINSTEIN, A. (2008): "The relationships between market orientation and alternative strategic orientations. A meta-analysis", European Journal of Marketing, Vol. 42, $\mathrm{N}^{\circ}$ 1/2, pp. 115-134.

HABER, S. Y REICHEL, A. (2005): "Identifying performance measures of small ventures-the case of the tourism industry", Journal of Small Business Management, Vol. 43, № 3, pp. 257-286.

HAIR, J.F., ANDERSON, R.E., TATHAM, R.L. Y BLACK, W.C. (1998): Multivariate data analysis, Upper Saddle River, New Jersey: Prentice-Hall.

HITT, M.A., IRELAND, R.D., CAMP, S.M. Y SEXTON, D.L. (2001): “Guest editors' introduction to special issue strategic entrepreneurship: entrepreneurial strategies for wealth creation", Strategic Management Journal, Vol. 22, pp. 479-491.

HUGHES, M. Y MORGAN, R.E. (2007): "Deconstructing the relationship between entrepreneurial orientation and business performance at the embryonic stage of firm growth", Industrial Marketing Management, Vol. 36, pp. 651-61.

HULT, G.T.M. Y KETCHEN, D.J. JR (2001): "Does market orientation matter? A test of the relationship between positional advantage and performance", Strategic Management Journal, Vol. 22, pp. 899-906.

HULT, G.T.M., HURLEY, R.F. Y KINGHT, G.A. (2004): "Innovativeness: its antecedents and impact on business performance", Industrial Marketing Management, Vol. 33, pp. 429-38.

HUNT, S.D. Y MORGAN, R.M. (1995): “The resource-advantage theory of competition: dynamics, path dependencies, and evolutionary dimensions", Journal of Marketing, Vol. 59, № 2, pp. 1-15.

HURLEY, R.F. Y HULT, G.T.M. (1998): "Innovation, market orientation and organizational learning: an integration and empirical examination", Journal of Marketing, Vol. 62, No 3, pp. 42-54.

JIMÉNEZ, D. Y CEGARRA, J.G. (2007): "The performance effect of organizational learning and market orientation", Industrial Marketing Management, Vol. 36, pp. 694-708.

KAKATI, M. (2003): "Success criteria in high-tech new ventures", Technovation, Vol. 23, pp. 447457.

KESKIN, H. (2006): "Market orientation, learning orientation and innovation capabilities in SMEs", European Journal of Innovation Management, Vol. 9, № 4, pp. 396-417.

KIRCA, A., JAYACHANDRAN, S.Y BEARDEN, W. (2005): "Market orientation: a meta-analytic review and assessment of its antecedents and impact on performance", Journal of Marketing, Vol. 69, pp. 24-41.

KOHLI, A. Y JAWORSKI, B.J. (1990): "Market orientation: the construct, research propositions and managerial implications", Journal of Marketing, Vol. 54, $\mathrm{N}^{\circ}$ 2, pp. 1-18.

KROPP, F., LINDSAY, N.J. Y SHOHAM, A. (2006): "Entrepreneurial, market, and learning orientations and international entrepreneurial business venture performance in South African firms", International Marketing Review, Vol. 23, №. 5, pp. 504-523

LAITINEN, E.K. (1992): "Prediction of failure of a newly founded firm", Journal of Business Venturing, Vol. 7, pp. 323-40.

LEE, C., LEE, K. Y PENNINGS, J.M. (2001): "Internal capabilities, external networks, and performance: a study of technology-based ventures", Strategic Management Journal, Vol. 22, pp. 615-640.

LI, H. Y ATUAHENE-GIMA, K. (2001): Product innovation strategy and the performance of new technology ventures in China, “Academy of Management Journal”, Vol. 44, № 6, pp. 1123-1134.

LUMPKIN, G.T Y DESS, G.G. (1996): "Claryfing the entrepreneurial orientation construct and linking it to performance", Academy of Management Review, Vol. 21, $\mathrm{N}^{\circ} 1$, pp. 135-72. 
LUMPKIN, G.T.Y DESS, G.G. (2001): "Linking two dimensions of entrepreneurial orientation to firm performance: the moderating role of environment and industry life cycle", Journal of Business Venturing, Vol. 16, pp. 429-51.

MAVONDO, F.T., CHIMHANZI, J. Y STEWART, J. (2005): "Learning orientation and market orientation. Relationship with innovation, human resource practices and performance" European Journal of Marketing, Vol. 39, $\mathrm{N}^{\circ}$ 11/12, pp. 1235-1263.

MINNITI, M. Y LÉVESQUE, M. (2008): "Recent developments in the economics of entrepreneurship", Journal of Business Venturing, Vol. 23, pp. 603-12.

NAMAN, J.L. Y SLEVIN, D.P. (1993): "Entrepreneurship and the concept of fit: a model and empirical tests", Strategic Management Journal, Vol. 14, pp. 137-53.

NARVER, J.C. Y SLATER, S.F. (1990): "The effect of a market orientation on business profitability", Journal of Marketing, Vol. 54, No 4, pp. 20-35.

NEWBERT, S.L., KIRCHOFF, B.A. Y WALSH, S.T. (2007): "Defining the relationship among founding resources, strategies, and performance in techonlogy-intensive new ventures: evidence from the semiconductor silicon industry", Journal of Small Business Management, Vol. 45, $\mathrm{N}^{\mathrm{o}}$ 4, pp. 438-66.

OSBORNE, R.L. (1993): "Why entrepreneurs fail: how to avoid the traps", Management Decision, Vol. 31, No 1, pp. 18-21.

PALADINO, A. (2007): "Investigating the drivers of innovation and new product success: a comparison of strategic orientations", The Journal of Product Innovation Management, Vol. 24, pp. 534-553.

PALADINO, A. (2008): "Analyzing the effects of market and resource orientations on innovative outcomes in times of turbulence", The Journal of Product Innovation Management, Vol. 24, pp. 534-553.

PELHAM, A.M. (1999): "Influence of environment, strategy, and market orientation on performance in small manufacturing firms”, Journal of Business Research, Vol. 45, pp. 33-46.

PELHAM, A.M. (2000): "Market orientation and other potential influences on performance in small and medium-sized manufacturing firms", Journal of Small Business Management, Vol. 38, $\mathrm{N}^{\circ}$ 1, pp. 48-67.

PELHAM, A. M. Y WILSON, D. T. (1996): “A longitudinal study of the impact of market structure, firm structure, strategy, and market orientation culture on dimension of small-firm performance", Journal of the Academy of Marketing Science, Vol. 24, pp. 27-43.

PETERAF, M. (1993): “The cornerstones of competitive advantage: a resourced based view", Strategic Management Journal, Vol. 14, $\mathrm{N}^{\mathrm{o}} 3$, pp. 57-83.

PORTER, M. (1980): Competitive Strategy, The Free Press, N.Y.

RAUCH, A., WIKLUND, J., LUMPKIN, G.T. Y FRESE, M. (2009): "Entrepreneurial orientation and business performance: an assessment of past research and suggestions for the future", Entrepreneurship Theory and Practice (Mayo), pp. 761-787.

RENKO, M., CARSRUD, A. Y BRÄNNBACK, M. (2009): “The effect of a market orientation, entrepreneurial orientation, and technological capability on innovativeness: a study of young biotechnology ventures in the United States and in Scandinavia”, Journal of Small Business Management, Vol. 47, $\mathrm{N}^{\circ} 3$, pp. 331-369.

ROSENBUSCH, N., BRINCKMANN, J. Y BAUSCH, A. (2010): "Is innovation always beneficial? A meta-analysis of the relationship between innovation and performance in SMEs", Journal of Business Venturing (En prensa).

RUNYAN, R., DROGE, C. Y SWINNEY, J. (2008): "Entrepreneurial orientation versus small business orientation: what are their relationships to firm performance?" Journal of Small Business Management, Vol. 46, $\mathrm{N}^{\mathrm{o}}$ 4, pp. 567-588.

SALAVOU, H. (2005): "Do customer and technology orientations influence product innovativeness in SMEs? Some evidence from Greece”, Journal of Marketing Management, Vol. 21, pp. 307-338. 
SANDVIK, I.L. Y SANDVIK, K. (2003): “The impact of market orientation on product innovativeness and business performance", International Journal of Research in Marketing, Vol. 20, pp. 355-376.

SANTOS, M.L., SANZO, M.J., ÁLVAREZ, L.I. Y VÁZQUEZ, R. (2005): “Organizational learning and market orientation: interface and effects on performance", Industrial Marketing Management, Vol. 34, pp. 187-202.

SCIASCIA, S., NALDI, L. Y HUNTER, E. (2006): "Market orientation as determinant of entrepreneurship: An empirical investigation on SMEs", Entrepreneurship Management, Vol. 2, pp. $21-38$.

SHANE, S. Y VENKATARAMAN, S. (2000): "The promise of entrepreneurship as a field of research", Academy of Management Review, Vol. 25, pp. 217-226.

SINKULA, J.M. (1994): "Market information processing and organizational learning", Journal of Marketing, Vol. 58, No 1, pp. 35-45.

SINKULA, J. M., BAKER, W. Y NOORDEWIER, T. G. (1997): “A Framework for market-based organizational learning: linking values, knowledge and behavior," Journal of the Academy of Marketing Science, Vol. 25 (Otoño), pp. 305-318.

SLATER, S.F. Y NARVER, J.C. (1995): "Market orientation and the learning organization", Journal of Marketing, Vol. 59, $\mathrm{N}^{\circ}$ 3, pp. 63-74.

SONG, M., PODOYNITSYNA, K., VAN DER BIJ, H. Y HALMAN, J.I.M. (2008): "Success factors in new ventures: a meta-analysis", The Journal of Product Innovation Management, Vol. 25, pp. 7-27.

STAM, W. Y ELFRING, T. (2008): "Entrepreneurial orientation and new venture performance: the moderating role of intra-and extra industry social capital", Academy of Management Journal, Vol. 51, Nº 1, pp. 97-111.

URBANO, D. (2006): La creación de empresas en Cataluña: organismos de apoyo y actitudes hacia la actitud emprendedora, Generalitat de Catalunya, Departament de treball i industria, Centre de innovació i desenvolupament empresarial (CIDEM), Barcelona.

VÁZQUEZ, R., SANTOS, M.L. Y ÁLVAREZ, L.I. (2001): "Market orientation, innovation and competitive strategies in industrial firms", Journal of Strategic Marketing, Vol. 9, pp. 69-90.

VERHEES, F.J.H.M. Y MEULENBERG, M.T.G. (2004): "Market orientation, innovativeness, product innovation and performance in small firms", Journal of Small Business Management, Vol. 42, No 4, pp. 134-154.

WANG, C.L. (2008): "Entrepreneurial orientation, learning orientation, and firm performance", Entrepreneurship Theory and Practice, (Julio), pp. 635-657.

WATSON, K., HOGARTH-SCOTT, S Y WILSON, N. (1998): "Small business start-ups: success factors and support implications", International Journal of Entrepreneurial Behaviour \& Research, Vol. 4, No 3, pp. 217-38.

WERNERFELT, B. (1984): “A resource-based view of the firm”, Strategic Management Journal, Vol. 5, $\mathrm{N}^{\circ}$ 2, pp. 171-180.

WIKLUND, J. (1999): "The sustainability of the entrepreneurial orientation-performance relationship", Entrepreneurship Theory and Practice, (Otoño), pp. 37-48.

WIKLUND, J. Y SHEPHERD, D. (2005): "Entrepreneurial orientation and small business performance: a configurational approach", Journal of Business Venturing, Vol. 20, pp. 71-91.

ZAHRA, S.A. Y COVIN, J. (1995): "Contextual influences on the corporate entrepreneurshipcompany performance relationship in established firms: A longitudinal analysis", Journal of Business Venturing, Vol. 10, pp. 43-58.

ZHOU, Z.K., YIM, K.C. Y TSE, D. (2005): "The effects of strategic orientations on technology- and market-based breakthrough innovations", Journal of Marketing, Vol. 69 No 1, pp. 42-60. 


\section{Anexo 1. ESCALA DE MEDICIÓN DE LA ORIENTACIÓN AL MERCADO}

Acuerdo-Desacuerdo

\begin{tabular}{|c|c|}
\hline $\begin{array}{l}\text { 1. Nuestras estrategias van dirigidas a conseguir más valor para nuestros } \\
\text { clientes }\end{array}$ & 1234567 \\
\hline 2. Fijamos periódicamente objetivos de satisfacción de los clientes & 1234567 \\
\hline 3. Respondemos rápidamente a las acciones de la competencia & 1234567 \\
\hline $\begin{array}{l}\text { 4. Hacemos un seguimiento permanente del nivel de compromiso en la } \\
\text { satisfacción de las necesidades de los clientes }\end{array}$ & 1234567 \\
\hline $\begin{array}{l}\text { 5. Los directivos de los distintos departamentos de nuestra empresa se } \\
\text { comunican regularmente con los clientes }\end{array}$ & 1234567 \\
\hline $\begin{array}{l}\text { 6. La información acerca de nuestros clientes circula por toda la empresa/ } \\
\text { división }\end{array}$ & 1234567 \\
\hline $\begin{array}{l}\text { 7. Para el logro de ventajas competitivas nos basamos sobre todo en el } \\
\text { conocimiento de las necesidades de los clientes }\end{array}$ & 1234567 \\
\hline $\begin{array}{l}\text { 8. Todas nuestras áreas funcionales actúan de manera coordinada para } \\
\text { satisfacer las necesidades de los clientes }\end{array}$ & 1234567 \\
\hline $\begin{array}{l}\text { 9. Nuestro equipo comercial intercambia regularmente información sobre } \\
\text { las actividades de la competencia }\end{array}$ & 1234567 \\
\hline $\begin{array}{l}\text { 10. Medimos de manera frecuente y sistemática el grado de satisfacción de } \\
\text { nuestros clientes }\end{array}$ & 1234567 \\
\hline $\begin{array}{l}\text { 11. Los ejecutivos analizamos y discutimos a menudo los puntos fuertes y } \\
\text { débiles de la competencia }\end{array}$ & 1234567 \\
\hline $\begin{array}{l}\text { 12. Nuestros directivos saben la manera de conseguir que todos los } \\
\text { empleados contribuyan a crear valor para los clientes }\end{array}$ & 1234567 \\
\hline $\begin{array}{l}\text { 13. Nos fijamos objetivos de captación de clientes en los mercados en que } \\
\text { disponemos de ventajas competitivas }\end{array}$ & 1234567 \\
\hline 14. Las distintas áreas funcionales comparten recursos entre sí & 12334567 \\
\hline
\end{tabular}




\section{Anexo 2. ESCALA DE MEDIDA DE LA ORIENTACIÓN AL APRENDIZAJE}

Acuerdo-Desacuerdo

1. Los administradores de nuestro negocio creemos que la habilidad de aprender es la clave para nuestra ventaja competitiva

12345567

2. Los valores clave de este negocio incluyen al aprendizaje como llave de mejora

3. Nosotros pensamos que el aprendizaje del empleado es una inversión, no un gasto

1223345667

4. Pensamos en esta empresa que si nos quedamos pasivos en nuestro aprendizaje, dañaremos nuestro futuro

12334567

5. Nuestra cultura establece que el aprendizaje organizativo no es prioritario (Invertida)

6. Los altos ejecutivos creemos conveniente compartir nuestra visión de negocio con todos los empleados

122345567

7. Todos los empleados estamos comprometidos con las metas de este negocio

8. Los empleados se ven a si mismos como socios y dan su mayor esfuerzo para mejorar el rumbo del negocio

12234567

9. Hay un total acuerdo con la visión del negocio a través de todos los niveles, funciones y áreas de la empresa

122334566

. La empresa apoya a los empleados a buscar nuevas maneras de hacer las cosas

12234567

1. Estamos abiertos está abiertos a críticas sobre la forma en que hacemos el trabajo

1223345667

2. El énfasis en la innovación constante no forma parte de nuestra cultura en la empresa (Invertida)

122345567

122345567

123345667

13. Ideas originales son altamente valoradas en esta organización

122345667

1223445667 


\section{Anexo 3. ESCALA DE MEDICIÓN DE LA ORIENTACIÓN EMPRENDEDORA}

Acuerdo-Desacuerdo

\begin{tabular}{|c|c|}
\hline $\begin{array}{l}\text { 1. Otorgamos más énfasis a la investigación y el desarrollo de nuevos pro- } \\
\text { ductos o servicios que a la comercialización de productos que el mercado } \\
\text { ya conoce }\end{array}$ & 1234567 \\
\hline $\begin{array}{l}\text { 2. En general, en la empresa tomamos proyectos con bajo riesgo y benefi- } \\
\text { cios normales en lugar de proyectos de alto riesgo con probabilidad de } \\
\text { altas tasas de beneficios (Invertida) }\end{array}$ & 1234567 \\
\hline $\begin{array}{l}\text { 3. En general, nosotros creemos en grandes cambios y rápidos, que en } \\
\text { cambios pequeños y lentos }\end{array}$ & 1234567 \\
\hline $\begin{array}{l}\text { 4. Nuestros cambios en los últimos años en las líneas de productos o servi- } \\
\text { cios del negocio han sido constantes e importantes }\end{array}$ & 1234567 \\
\hline $\begin{array}{l}\text { 5. Nuestra empresa en lugar de tener acciones pioneras en el mercado típica- } \\
\text { mente responde a acciones que los competidores han iniciado (Invertida) }\end{array}$ & 1234567 \\
\hline $\begin{array}{l}\text { 6. Nuestra empresa típicamente adopta medidas agresivas para "eliminar" a } \\
\text { los competidores del mercado, en lugar de tomar una postura de "vive y } \\
\text { deja vivir" }\end{array}$ & $\begin{array}{lllllll}1 & 2 & 3 & 4 & 5 & 6 & 7\end{array}$ \\
\hline
\end{tabular}

\section{Anexo 4. ESCALA DE MEDICIÓN DE LA INNOVACIÓN}

Inferior-Superior

\begin{tabular}{|l|l|l|}
\hline $\begin{array}{l}\text { 1. La tasa de nuevos productos o servicios en nuestra empresa en compara- } \\
\text { ción con la tasa de nuestros competidores directos es }\end{array}$ & 1234567 \\
\hline $\begin{array}{l}\text { 2. El grado de diferenciación entre las innovaciones nuestras y las innova- } \\
\text { ciones de nuestros competidores directos es }\end{array}$ & 1234567 \\
\hline $\begin{array}{l}\text { 3. La tasa de éxito de nuevos productos en relación a la tasa de nuestros } \\
\text { competidores directos es }\end{array}$ & 1234567 \\
\hline
\end{tabular}

\section{Anexo 5. ESCALA DE MEDICIÓN DE LOS RESULTADOS ORGANIZATIVOS}

\begin{tabular}{|c|c|}
\hline & Inferior-Superior \\
\hline $\begin{array}{l}\text { 1. En relación a sus objetivos, el nivel de rentabilidad sobre la inversión } \\
\text { (ROI) en el último año fue }\end{array}$ & 1234567 \\
\hline 2. En relación a sus objetivos, el nivel de beneficios en el último año fue & 12334567 \\
\hline $\begin{array}{l}\text { 3. En relación a sus objetivos, el nivel de incremento en sus ventas en el } \\
\text { último año fue }\end{array}$ & 1234567 \\
\hline $\begin{array}{l}\text { 4. En relación a sus objetivos, el grado de satisfacción de sus clientes en el } \\
\text { último año fue }\end{array}$ & 1234567 \\
\hline $\begin{array}{l}\text { 5. En relación a sus objetivos, la satisfacción de los empleados en el trabajo } \\
\text { en el último año fue }\end{array}$ & 1234567 \\
\hline $\begin{array}{l}\text { 6. En relación a sus objetivos, los resultados globales en su empresa en el } \\
\text { último año fueron }\end{array}$ & 1234567 \\
\hline
\end{tabular}




\section{Anexo 6. RESULTADOS DEL EFA}

\begin{tabular}{|c|c|c|c|c|c|c|c|c|}
\hline & \multicolumn{2}{|c|}{ EFA } & \multicolumn{4}{|c|}{ EFA } & \multicolumn{2}{|c|}{ EFA } \\
\hline $\begin{array}{c}\text { Orientación al } \\
\text { cliente }\end{array}$ & $\begin{array}{l}\text { Factor } \\
\text { loadings }\end{array}$ & $\begin{array}{l}\text { Item-to } \\
\text { total } \\
\text { correlation }\end{array}$ & $\begin{array}{c}\text { Orientación } \\
\text { a la } \\
\text { competencia }\end{array}$ & $\begin{array}{c}\text { Factor } \\
\text { loadings }\end{array}$ & $\begin{array}{c}\text { Item-to } \\
\text { total } \\
\text { correlation }\end{array}$ & $\begin{array}{l}\text { Coordinación } \\
\text { interfuncional }\end{array}$ & $\begin{array}{c}\text { Factor } \\
\text { loadings }\end{array}$ & $\begin{array}{c}\text { Item-to } \\
\text { total } \\
\text { correlation }\end{array}$ \\
\hline OCLI_1 & 0,72 & $0,708 * * *$ & OCOM_1 & 0,85 & $0,857 * * *$ & CFUN_1 & 0,79 & $0,789 * * *$ \\
\hline OCLI_2 & 0,78 & $0,772 * * *$ & OCOM_2 & 0,78 & $0,804 * * *$ & CFUN_2 & 0,85 & $0,851 * * *$ \\
\hline OCLI_3 & 0,78 & $0,779 * * *$ & OCOM_3 & 0,88 & $0,866 * * *$ & CFUN_3 & 0,82 & $0,826 * * *$ \\
\hline OCLI_4 & 0,67 & $0,695 * * *$ & OCOM_4 & 0,86 & $0,839 * * *$ & CFUN_4 & 0,84 & $0,838 * * *$ \\
\hline OCLI_5 & 0,75 & $0,755 * * *$ & & & & & & \\
\hline OCLI_6 & 0,81 & $0,808 * * *$ & & & & & & \\
\hline KMO & \multicolumn{2}{|c|}{0,791} & KMO & \multicolumn{2}{|c|}{0,815} & $\mathrm{KMO}$ & \multicolumn{2}{|c|}{0,812} \\
\hline Barlett & \multicolumn{2}{|c|}{$501,918 * * *$} & Barlett & \multicolumn{2}{|c|}{$379,199 * * *$} & Barlett & \multicolumn{2}{|c|}{$322,457 * * *$} \\
\hline$\%$ acc & \multicolumn{2}{|c|}{$56,89 \%$} & $\%$ acc & \multicolumn{2}{|c|}{$71,04 \%$} & $\%$ acc & \multicolumn{2}{|c|}{$68,32 \%$} \\
\hline Cronbach $\alpha$ & \multicolumn{2}{|c|}{0,846} & Cronbach $\alpha$ & \multicolumn{2}{|c|}{0,857} & Cronbach $\alpha$ & \multicolumn{2}{|c|}{0,845} \\
\hline $\begin{array}{l}\text { Compromiso } \\
\text { con el } \\
\text { aprendizaje }\end{array}$ & $\begin{array}{l}\text { Factor } \\
\text { loadings }\end{array}$ & $\begin{array}{c}\text { Item-to } \\
\text { total } \\
\text { correlation }\end{array}$ & $\begin{array}{c}\text { Visión } \\
\text { compartida }\end{array}$ & $\begin{array}{l}\text { Factor } \\
\text { loadings }\end{array}$ & $\begin{array}{c}\text { Item-to } \\
\text { total } \\
\text { correlation }\end{array}$ & $\begin{array}{c}\text { Mentalidad } \\
\text { abierta }\end{array}$ & $\begin{array}{c}\text { Factor } \\
\text { loadings }\end{array}$ & $\begin{array}{c}\text { Item-to } \\
\text { total } \\
\text { correlation }\end{array}$ \\
\hline CAPR_1 & 0,83 & $0,829 * * *$ & VCOM_1 & 0,85 & $0,845 * * *$ & MAB_1 & 0,86 & $0,857 * * *$ \\
\hline CAPR_2 & 0,90 & $0,893 * * *$ & VCOM_2 & 0,91 & $0,911 * * *$ & MAB_2 & 0,88 & $0,876 * * *$ \\
\hline CAPR_3 & 0,84 & $0,842 * * *$ & VCOM_3 & 0,90 & $0,910 * * *$ & MAB_3 & 0,82 & $0,826 * * *$ \\
\hline CAPR_4 & 0,88 & $0,882 * * *$ & VCOM_4 & 0,87 & $0,870 * * *$ & MAB_4 & 0,83 & $0,832 * * *$ \\
\hline CAPR_5 & 0,77 & $0,783 * * *$ & & & & & & \\
\hline $\mathrm{KMO}$ & \multicolumn{2}{|c|}{0,87} & $\mathrm{KMO}$ & \multicolumn{2}{|c|}{0,829} & $\mathrm{KMO}$ & \multicolumn{2}{|c|}{0,79} \\
\hline Barlett & \multicolumn{2}{|c|}{$619,156 * * *$} & Barlett & \multicolumn{2}{|c|}{$536,94 * * *$} & Barlett & \multicolumn{2}{|c|}{$404,964 * * *$} \\
\hline$\%$ acc & \multicolumn{2}{|c|}{$71,67 \%$} & $\%$ acc & \multicolumn{2}{|c|}{$78,24 \%$} & $\%$ acc & \multicolumn{2}{|c|}{$71,90 \%$} \\
\hline Cronbach $\alpha$ & \multicolumn{2}{|c|}{0,9} & Cronbach $\alpha$ & \multicolumn{2}{|c|}{0,906} & Cronbach $\alpha$ & \multicolumn{2}{|c|}{0,869} \\
\hline $\begin{array}{l}\text { Orientación } \\
\text { emprendedora }\end{array}$ & $\begin{array}{l}\text { Factor } \\
\text { loadings }\end{array}$ & $\begin{array}{c}\text { Item-to } \\
\text { total } \\
\text { correlation }\end{array}$ & Innovación & $\begin{array}{l}\text { Factor } \\
\text { loadings }\end{array}$ & $\begin{array}{c}\text { Item-to } \\
\text { total } \\
\text { correlation }\end{array}$ & $\begin{array}{c}\text { Resultados } \\
\text { organizativos }\end{array}$ & $\begin{array}{l}\text { Factor } \\
\text { loadings }\end{array}$ & $\begin{array}{c}\text { Item-to } \\
\text { total } \\
\text { correlation }\end{array}$ \\
\hline OEMP_1 & 0,74 & $0,74 * * *$ & INOV_1 & 0,91 & $0,906 * * *$ & ROI_S & 0,83 & $0,831 * * *$ \\
\hline OEMP_2 & 0,74 & $0,738 * * *$ & INOV_2 & 0,91 & $0,919 * * *$ & BFC_S & 0,89 & $0,888 * * *$ \\
\hline OEMP_3 & 0,82 & $0,812 * * *$ & INOV_3 & 0,90 & $0,894 * * *$ & VTAS_S & 0,87 & $0,873 * * *$ \\
\hline OEMP_4 & 0,82 & $0,829 * * *$ & & & & R_SCLI & 0,68 & $0,690 * * *$ \\
\hline OEMP_5 & 0,80 & $0,807 * * *$ & & & & R_SEMP & 0,73 & $0,737 * * *$ \\
\hline OEMP_6 & 0,85 & $0,848 * * *$ & & & & R_GLOB & 0,89 & $0,886 * * *$ \\
\hline KMO & & 891 & KMO & & 748 & KMO & & 864 \\
\hline Barlett & 589. & $78 * * *$ & Barlett & 348 & $88 * * *$ & Barlett & 803 & $82 * * *$ \\
\hline$\%$ acc & &, $5 \%$ & $\%$ acc & & $18 \%$ & $\%$ acc & & $44 \%$ \\
\hline Cronbach $\alpha$ & & 884 & Cronbach $\alpha$ & &, 89 & Cronbach $\alpha$ & & 901 \\
\hline
\end{tabular}

$* * *$ sig. $<0,01$ 


\section{Anexo 7. RESULTADOS DEL CFA}

\begin{tabular}{|c|c|c|c|c|c|}
\hline & & & Standarized Lambda & AVE & $\mathrm{CR}$ \\
\hline OCLI_1 & $<---$ & Ocl & 0,62 & \multirow[t]{6}{*}{0,50} & \multirow[t]{6}{*}{0,80} \\
\hline OCLI_2 & $<---$ & Ocl & 0,71 & & \\
\hline OCLI_3 & $<---$ & Ocl & 0,76 & & \\
\hline OCLI_4 & $<---$ & Ocl & 0,61 & & \\
\hline OCLI_5 & $<---$ & Ocl & 0,75 & & \\
\hline OCLI_6 & $<---$ & Ocl & 0,76 & & \\
\hline OCOM_1 & $<---$ & Oco & 0,76 & \multirow[t]{4}{*}{0,63} & \multirow[t]{4}{*}{0,8} \\
\hline OCOM_2 & $<---$ & Oco & 0,74 & & \\
\hline OCOM_3 & $<---$ & Oco & 0,79 & & \\
\hline OCOM_4 & $<---$ & Oco & 0,89 & & \\
\hline CFUN_1 & $<<--$ & Cint & 0,72 & \multirow[t]{4}{*}{0,58} & \multirow[t]{4}{*}{0,85} \\
\hline CFUN_2 & $<---$ & Cint & 0,82 & & \\
\hline CFUN_3 & $<---$ & Cint & 0,76 & & \\
\hline CFUN_4 & $<---$ & Cint & 0,73 & & \\
\hline CAPR_1 & $<---$ & Cap & 0,76 & \multirow[t]{5}{*}{0,64} & \multirow[t]{5}{*}{0,90} \\
\hline CAPR_2 & $<---$ & Cap & 0,85 & & \\
\hline CAPR_3 & $<---$ & Cap & 0,80 & & \\
\hline CAPR_4 & $<---$ & Cap & 0,86 & & \\
\hline CAPR_5 & $<---$ & Cap & 0,72 & & \\
\hline VCOM_1 & $<---$ & VCo & 0,75 & \multirow[t]{4}{*}{0,73} & \multirow[t]{4}{*}{0,92} \\
\hline VCOM_2 & $<---$ & VCo & 0,95 & & \\
\hline VCOM_3 & $<---$ & VCo & 0,84 & & \\
\hline VCOM_4 & $<---$ & $\mathrm{VCo}$ & 0,88 & & \\
\hline MAB_1 & $<---$ & Mab & 0,79 & \multirow[t]{4}{*}{0,61} & \multirow[t]{4}{*}{0,86} \\
\hline MAB_2 & $<---$ & Mab & 0,80 & & \\
\hline MAB_3 & $<---$ & Mab & 0,78 & & \\
\hline MAB_4 & $<---$ & Mab & 0,75 & & \\
\hline OEMP_1 & $<---$ & $\mathrm{OE}$ & 0,67 & \multirow[t]{6}{*}{0,56} & \multirow[t]{6}{*}{0,89} \\
\hline OEMP_2 & $<---$ & $\mathrm{OE}$ & 0,64 & & \\
\hline OEMP_3 & $<---$ & $\mathrm{OE}$ & 0,76 & & \\
\hline OEMP_4 & $<---$ & $\mathrm{OE}$ & 0,80 & & \\
\hline OEMP_5 & $<---$ & $\mathrm{OE}$ & 0,77 & & \\
\hline OEMP_6 & $<---$ & $\mathrm{OE}$ & 0,85 & & \\
\hline INOV_1 & $<--$ & CI & 0,85 & \multirow[t]{3}{*}{0,73} & \multirow[t]{3}{*}{0,89} \\
\hline INOV_2 & $<---$ & CI & 0,88 & & \\
\hline INOV_3 & $<---$ & $\mathrm{CI}$ & 0,84 & & \\
\hline ROI_S & $<---$ & RO & 0,83 & \multirow[t]{6}{*}{0,63} & \multirow[t]{6}{*}{0,91} \\
\hline BFC_S & $<---$ & RO & 0,90 & & \\
\hline VTAS_S & $<---$ & RO & 0,85 & & \\
\hline R_SCLI & $<---$ & $\mathrm{RO}$ & 0,66 & & \\
\hline R_SEMP & $<---$ & RO & 0,63 & & \\
\hline R_GLOB & $<---$ & RO & 0,87 & & \\
\hline
\end{tabular}

$\chi^{2}=1277,735 * * * ; \chi^{2} / \mathrm{df}=1.666$

$C F I=0,913$

RMSEA $=0,057($ LO $90=0,052 ;$ HI $90=0,063)$

$* * *$ sig. $<0,01$ 